\title{
Evaluation of Serum Apelin-13 in Acne Vulgaris Patients
}

N.E.Sorour ${ }^{1}$, N.A.Abdalhafeez ${ }^{2}$, E.M.Akl ${ }^{1}$ and M.A.bd El-wahed ${ }^{1}$

${ }^{1}$ Dermatology, Venereology and Andrology Dept., Faculty of Medicine, Benha Univ.,Benha, Egypt

${ }^{2}$ Clinical Pathology and Immunology Dept., Faculty of Medicine, Benha Univ.,Benha,Egypt

E-Mail: monaarafatrezq@gmail.com

\begin{abstract}
Acne is a longstanding inflammatory disease of the pilosebaceous unit resulting from androgen-induced increased sebum production, altered keratinization, inflammation, and bacterial colonization of hair follicles by Propionibacterium acne [1]. Apelin is a newely discovered adipocytokine secreted by white adipose tissue and couples with the APJ receptor with the highest affinity. Apelin and the APJ receptor are found in pancreatic islet cells and found to have a role in energy metabolism. Many researches show that apelin has a role in glucose homeostasis regulation, insulin secretion, and increasing insulin sensitivity [2].The aim of the work was to evaluate the serum level of Apelin-13 in patients with acne vulgaris .Serum apelin was measured in 40 patients with moderate to severe acne vulgaris and 30 healthy controls using enzyme-linked immunosorbent assay (ELISA). Serum levels of apelin13 in patients with acne vulgaris was found to be higher than in healthy controls ( $p$ value $<0.005$ ). In Conclusion Serum apelin13 has a role in the pathophysiology of acne vulgaris patients.
\end{abstract}

Keywords: Acne vulgaris, Acne patients, Apelin13.

\section{Introduction}

The prevalence of acne vulgaris is $85 \%$ of the adolescent population, the sites with a higher density of sebaceous glands have been more affected such as the face, back, and chest [3].

The main pathogenic factors of acne are high sebum secretion, follicular hyperkertinization, high androgen actions, propionibacterium acnes, colonization [4].

Apelin is an adipocytokine which has a role in glucose homeostasis and may contribute to the association between adipose tissue mass and metabolic diseases [5] .

The aim of this study was to evaluate the possible role of serum apelin 13 in the pathogenesis of acne vulgaris by measuring its level (by using ELISA) in the serum of acne patients and to correlate its level with the disease severity.

\section{Patients and methods}

This is a cross Section case control study. The study included forty patients with acne vulgaris and thirty healthy, age and sex matched controls attending the dermatology outpatient clinic at Benha University Hospital, Benha, Egypt from Janurary 2018 to Janurary 2019. Participants gave their informed written consent before enrollment and the study was approved by the Research Ethics Committee in Faculty of Medicine, Benha University.

All patients included in the study have moderate to severe recurrent acne lesions..Patients with skin disease other than acne and subjects with acute or chronic infections, malignancies, autoimmune disorders, hepatic or renal diseases were excluded. None of the patients was on systemic or topical treatment for one month before the study.
Disease severity was evaluated by Gobal acne grading system score (GAGS) [6]. Acne was diagnosed clinically.

\subsection{Laboratory investigations}

Five mls of venous blood were withdrawn from each patient and control subjects. The blood samples were allowed to clot completely (within 2hours) at the room temperature. Centrifugation was done at $1000 \times \mathrm{g}$. for 20 minutes to separate the serum. After centrifugation, serum was separated by a pipette and kept in one eppendorf tube labeled with the number of the person. Specimens were kept at $-20{ }^{\circ} \mathrm{C}$ to preserve until the time of the run, we avoided repeated freezethaw cycles. Serum apelin13 levels was determined using Enzyme-linked Immunosorbent Assay Kit For Apelin 13 provided by CLOUDCLONECORP/Houston\TXIUSA. according to the manufacturer's instructions.

\subsection{Statistical analysis}

Statistical analysis was performed using the Statistical Package for Social Sciences (SPSS) vs.25.

\section{Results}

\subsection{Clinical and demographic characteristics of} the studied group

This study included 70 subjects, 40 acne patients and 30 normal subjects as a control. There was no significant difference between both studied groups regarding age and gender Table $(1,2)$.

\subsection{Mean serum levels of Apelin 13 in patients and controls}

Regrading comparsion between patients and controls regrading serum level of apelin13, There was a statistically significant difference between. 
patients and control groups regarding apelin

level (P.value $<0.05)$ Table (3).

Table (1) Comparison between patients and controls as regard age

\begin{tabular}{lcccccc}
\hline & \multicolumn{2}{c}{ Patients (No.=40) } & \multicolumn{2}{c}{ Controls (No.=30) } & T & P-value \\
\cline { 2 - 7 } Age (years) & Mean & SD & Mean & SD & 1.475 & 0.144 \\
& 19 & \pm 3 & 20 & \pm 2 & & \\
\hline
\end{tabular}

Table (2) Comparison between patients and controls as regard sex

\begin{tabular}{|c|c|c|c|c|c|c|c|}
\hline & \multicolumn{2}{|c|}{ Patients $($ No. $=40)$} & \multicolumn{2}{|c|}{ Controls $($ No. $=\mathbf{3 0})$} & \multirow[t]{2}{*}{$\mathbf{X}^{2}$} & \multirow[t]{2}{*}{ P-value } \\
\hline & & No. & $\%$ & No. & $\%$ & & \\
\hline Sex & male & 28 & 63.3 & 14 & 46.7 & 2.27 & 0.131 \\
\hline & female & 12 & 36.7 & 16 & 53.3 & & \\
\hline
\end{tabular}

Table (3) Mean serum levels of Apelin13 in patients and controls

\begin{tabular}{llclccc}
\hline & \multicolumn{2}{c}{ Patients (No.=40) } & \multicolumn{2}{l}{ Controls (No.=30) } & T & P-value \\
\cline { 2 - 6 } Apelin $(\mathbf{n g} / \mathbf{m l})$ & Mean & SD & Mean & SD & 2.6 & $<0.05$ \\
& 610.3 & 150 & 321.2 & 120.8 & 2 & \\
\hline
\end{tabular}

\section{Discussion}

Acne is a chronic inflammatory disease of the pilosebaceous unit. The main pathogenic factors of acne are high sebum secretion, follicular hyperkertinization, high androgen actions and propionibacterium acnes, colonization [7].

Apelin, an endogenous ligand of the G-proteincoupled receptor, is a novel myokine and may play a key role in regulating energy metabolism [8].

To achieve this goal, this study was conducted on 70 subjects; 30 normal subject with no chronic inflammatory conditions and 40 patients with acne.

In this study the mean age of patients was $19 \pm 3$ years old as acne begins in the early teens with the onset of facial sebum production and facial comedones followed by inflammatory lesions [9].

Family history was more frequent in the group with AV with prevalence of $73.3 \%$, A study, with 1002 young Iranians, showed that AV risk doubled for those with a positive family history [10].

Acne was associated with a family history of acne in first-degree relatives (odds ratio 3.41, 95\% confidence interval 2.31-5.05). The risk of acne vulgaris was less in population with lower body mass index with a mor effect in male patients compared with female patients[11].

In this study the mean serum apelin13 level in acne patients was high in comparison with controls.

Apelin13 may have a role on pathogenesis of acne through two pathways. The first one is central pathway through increasing LH level that in turn increase the level of androgen level .The second through its relation with hyperinsulinemia and its relation to IGF -1, which is a contributing factor for acne via hyperkeratinization.

\section{Conclusion}

Serum apelin13 has a role in the pathophysiology of acne vulgaris patients.

\section{References}

[1] N. N. Schommer, R. L. Gallo: Structure and function of the human skin microbiome. Trends in microbiology, Vol, 21(12),PP. 660-668, 2013.

[2] H. Hu , L. He, L. Li and L. Chen : Apelin/APJ system as a therapeutic target in diabetes and its complications. Molecular genetics and metabolism,Vol. 119(1-2), PP.2027,2016.

[3] M. Picardo, L.F. Eichenfield, J . Tan :Acne and Rosacea. Dermatol Therapy (Heidelb).Vol. 7(1),PP.43-52,2017.

[4] N. Emiroğlu, F.P. Cengiz and F. Kemeriz : Insulin resistance in severe acne vulgaris. Postepy Dermatol Alergol.Vol. 32(4),PP.281$285,2015$.

[5] J. Krist, K. Wieder , N. Klöting , A. Oberbach , S.Kralisch and T. Wiesner et al :Effects of weight loss and exercise on apelin serum concentrations and adipose tissue expression in human obesity.Obes Facts Vol.6(1),PP.5769,2013.

[6] A. Doshi, , A.Zaheer and M.J.Stiller, M. J:A comparison of current acne grading systems and proposal of a novel system. International journal of dermatology.Vol. 36(6),PP. 416418,1997.

[7] E.A.Tanghetti : The role of inflammation in the pathology of acne. The Journal of clinical and aesthetic dermatology, Vol.6(9),PP. 27,2013.

[8] W.Ji, L. Gong, J. Wang, H. He, and Y. Zhang: Hypoxic exercise training promotes apelin/APJ expression in skeletal muscles of 
high fat diet-induced obese mice. Protein and peptide letters, Vol.24(1), PP.64-70,2017.

[9] H.C. Williams, R.P.Dellavalle and S. Garner : Acne vulgaris.Lancet ; Vol.379,PP.361372,2013.

[10] S.Z.Ghodsi , H. Orawa , C.C. Zouboulis : Prevalence, severity, and severity risk factors of acne in high school pupils: a community-based study. Journal of Investigation Dermatolology ;Vol.129,PP.2136-2141.

[11] A.Di Landro, S. Cazzaniga, F. Parazzini, V. Ingordo, F.Cusano, L. Atzori, et al : Family history, body mass index, selected dietary factors, menstrual history, and risk of moderate to severe acne in adolescents and young adults. Journal of the American Academy of Dermatology, Vol. 67(),PP.1129-1131,2012. 